ISSN 1995-5464 (Print); ISSN 2408-9524 (Online)

https://doi.org/10.16931/1995-5464.2020239-48

Трудности компьютерно-томографической Аиагностики гепаточемююлярного рака. Аекшия и Аемонстрашия наблюАений

\author{
Розенгауз E.B. ${ }^{1,2 *}$, Караханова А.Г. ${ }^{3}$ \\ ${ }^{1}$ ФГБУ "Российский научный центр радиологии и хирургических технологий имени академика \\ А.М. Гранова” Минздрава РФ; 197758, Санкт-Петербург, пос. Песочный, ул. Ленинградская, д. 70, \\ Российская Федерация \\ ${ }_{2}^{2}$ ФББОУ ВО “Северо-Западный государственный медицинский университет имени И.И. Мечникова” \\ Минздрава РФ; 191015, Санкт-Петербург, ул. Кирочная, д. 41, Российская Федерация \\ ${ }^{3}$ ФГБУ“"Национальный медицинский исследовательский центр онкологии имени Н.Н. Петрова" МЗ РФ; \\ 197758, Санкт-Петербург, пос. Песочный, д. 68, Российская Федерация
}

\begin{abstract}
Гепатоцеллюлярный рак занимает особое место в ряду злокачественных опухолей, поскольку компьютернотомографическая или магнитно-резонансная картина позволяет с большой точностью диагностировать заболевание без биопсии. Это обстоятельство диктует необходимость тщательной оценки классического и атипичных вариантов изменений, выявляемых при лучевой диагностике. В работе проиллюстрированы различные варианты КТ-картины гепатоцеллюлярного рака.
\end{abstract}

Ключевые слова: печень, гепатоцеллюлярный рак, компьютерная томография, контрастное усиление, паттерн контрастирования, цирроз, портальный тромбоз

Ссылка для цитирования: Розенгауз Е.В., Караханова А.Г. Трудности компьютерно-томографической диагностики гепатоцеллюлярного рака. Лекция и демонстрация наблюдений. Анналы хирургической гепатологии. 2020; 25 (2): 39-48. https://doi.org/10.16931/1995-5464.2020239-48.

Авторы подтверждают отсутствие конфликта интересов.

\title{
Challenges of hepatocellular cancer diagnosing in computed tomography. Lecture and demonstration of observations
}

\author{
Rozengauz E.V. ${ }^{1,2 *}$, Karakhanova A.G. ${ }^{3}$ \\ ${ }^{1}$ Granov Russian Scientific Center of Radiology and Surgical Technologies; 70, Leningradskaya str., Pesochny, \\ 197758, S.-Petersburg, Russian Federation \\ ${ }^{2}$ North-Western State Medical University named after I.I. Mechnikov; 41, Kirochnaya str., St. Petersburg, \\ 191050, Russian Federation \\ ${ }^{3}$ Petrov National Medical Research Center of Oncology; 68, Leningradskaya str., Pesochny, St. Petersburg, \\ 197758, Russian Federation
}

\begin{abstract}
Hepatocellular carcinoma has a special place among the malignant tumors, because the computed tomography or magnetic resonance picture allows to accurately disease diagnose without a biopsy. This circumstance calls for thorough assessment of the classical and atypical variants of changes detected during radiologic imaging. This paper illustrates various versions of the CT-scan picture of hepatocellular cancer. The various variants of a CT scan of hepatocellular carcinoma are illustrated in this publication.
\end{abstract}

Keywords: liver, hepatocellular carcinoma, computed tomography, contrast enhancement, enhancement pattern, cirrhosis, portal thrombosis

For citation: Rozengauz E.V., Karakhanova A.G. Challenges of hepatocellular cancer diagnosing in computed tomography. Lecture and demonstration of observations. Annaly khirurgicheskoy gepatologii = Annals of HPB surgery. 2020; 25 (2): 39-48. (In Russian). https://doi.org/10.16931/1995-5464.2020239-48.

No conflict of interests to declare. 


\section{Введение}

Диагностика гепатоцеллюлярного рака (ГЦР) базируется на клинических, лабораторных и инструментальных методах обследования. При этом основную информационную ценность представляют изобразительные методы диагностики: УЗИ - нативное и с контрастным усилением, КТ, МРТ, ПЭТ и ПЭТ-КТ [1, 2]. Диагностические исследования, выполняемые на фоне введения контрастного препарата в артерию (КТ-артериография и КТ-артериопортография), имеют более узкие показания и меньшее распространение.

Обнаружение патологического образования основывается на выявлении существующей или созданной искусственно с помощью контрастного усиления разницы между сигнальными характеристиками (эхогенностью, денситометрической плотностью, интенсивностью сигнала, степенью накопления радиофармпрепарата) нормальной паренхимы и патологического образования [3]. Распознавание нозологической природы процесса основывается на комплексе лучевых симптомов: характере контуров, массэффекте, гомогенности контрастирования, выявлении капсулы и имеющей наибольшее диагностическое значение оценке динамики накопления и выведения контрастного вещества (паттерн) во время многофазного исследования при однократном болюсном его введении [2-4].

В настоящее время сформировалось представление о возможностях указанных методов в диагностике ГЦР. Большинство авторов отдают предпочтение МРТ (чувствительность в 95\% ДИ $0,77-0,90$, специфичность $0,84-0,97)$, отмечают недостаточные операционные характеристики УЗИ и необходимость дальнейшего изучения многообещающего КУУЗИ, высокую частоту ложноотрицательных результатов ПЭТ-КТ. В практической работе большинство исследований печени выполняют с помощью КТ (чувствительность в 95\% ДИ 0,63-0,76, специфичность $0,87-0,98)[3]$.

Оценка паттерна контрастирования патологического образования имеет особую важность, поскольку при выявлении его типичного варианта во время лучевого исследования стратегия лечения может быть определена без морфологического подтверждения. Однако типичный, или “классический”, паттерн выявляют лишь немногим чаще $(56,4 \%)$, чем атипичный [5]. Множество морфологических вариантов строения и степени дифференцировки опухоли, разное содержание в ней жира, осложнения течения заболевания обусловливают различные изменения "классического" паттерна контрастирования [6-8]. Атипичные паттерны контрастирования опухоли затрудняют неинвазивную диагностику природы заболевания.
Цель публикации - иллюстрация различных паттернов контрастирования ГЦР и анализ причин их формирования.

\section{Материал и методы}

У всех пациентов сканирование выполняли, как правило, по стандартной методике: в нативную, артериальную (20-25 с), портальную (4560 с) и отсроченную (300 с) фазы на 64- и 320-срезовых томографах, толщина среза 1 мм. При необходимости получить информацию о венах печени исследование дополняли венозной (90110 с) фазой. Контрастный препарат с концентрацией йода 270-350 мг/мл вводили двухколбовым инжектором со скоростью 3-5 мл/с. Автоматический запуск сканирования осуществляли с помощью программы "SureStart" при достижении плотности в аорте +180 HU. Bce представленные наблюдения морфологически верифицированы.

\section{Результаты}

Нативная фаза многофазного исследования для диагностики ГЦР малоинформативна. Обычно опухолевые образования отображаются в ней как низко- или изоплотностные паренхиме очаги, гомогенные при малых размерах опухоли, гетерогенные - при крупных размерах. Типичным, или “классическим”, паттерном накопления считали значимое усиление опухоли в артериальную фазу с вымыванием контрастного вещества в портальную, венозную или отсроченную фазу (рис. 1). Вымыванием считали состояние, когда плотность опухоли становится меньше плотности окружающей паренхимы печени. Отсутствие изменений плотности участка опухоли при многофазном исследовании считали признаком некроза.

В большинстве наблюдений (до 90\%) ГЦР развивается на фоне предшествовавшего цирроза, и при его наличии типичный паттерн контрастирования имеет высокую (95-96\%) специфичность. Согласно рекомендациям EASL-EORTC [9] критерии диагностики должны быть следующими: при опухоли $>2$ см достаточно одного диагностического теста, показавшего типичный паттерн контрастирования. При образовании 1-2 см рекомендовано подтверждение двумя диагностическими тестами. Большое значение имеет уровень альфа-фетопротеина. Сочетание этих факторов позволяет, по принятым рекомендациям, выполнять хирургическое вмешательство без дооперационной морфологической верификации [10]. При обнаружении опухоли $<1$ см рекомендуют наблюдение с помощью УЗИ каждые 4 мес в первый год, позднее - каждые 6 мес.

Типичное наблюдение иллюстрирует рис. 2. Больной был оперирован без предварительной 
морфологической верификации, гистологический диагноз - умеренно дифференцированный ГЦР.

ГЦР имеет три основных макроскопических варианта: узловой (одноузловой, рис. 2, 3, 6 и многоузловой - порядка $20 \%$ наблюдений, рис. 8), гигантский (рис. 4,5$)$, инфильтративный (рис. 7). Каждый из морфологических вариантов может сопровождаться внутриорганными метастазами (рис. 9). С увеличением размера образования появляется негомогенность контрастирования (мозаичный паттерн, см. рис. 3, 4). При этом периферические отделы опухоли демонстрируют типичную последовательность накопления-вымывания контрастного препарата, центральные, за счет некроза, не изменяют плотности во время исследования (см. рис. 3).

Диагностическое и прогностическое значение имеет обнаружение капсулы. Она выглядит как ободок низкой плотности вокруг опухоли (“хало”, см. рис. 3 д) или накапливающий кон- трастное вещество на более поздних отсроченных фазах контрастирования (см. рис. 5 б). Капсула ассоциируется с более благоприятным прогнозом, отсутствие капсулы (см. рис. 7) с неблагоприятным прогнозом [6].

Паттерн контрастирования опухоли зависит также от степени ее морфологической дифференцировки. При сравнении отмечена меньшая частота гиперваскулярности в артериальную фазу и тенденция к более раннему вымыванию контрастного вещества у низкодифференцированных опухолей по сравнению с высокодифференцированными $[5,8,11]$, что иллюстрирует рис. 4. Для низкодифференцированного ГЦР также характерно наличие геморрагий, зон некроза, кистозной дегенерации, внутриопухолевых сосудов и аневризм. По данным группы авторов [5], внутриопухолевые аневризмы были выявлены у 13 из 14 больных низкодифференцированным ГЦР. Собственное наблюдение приведено на рис. 5.
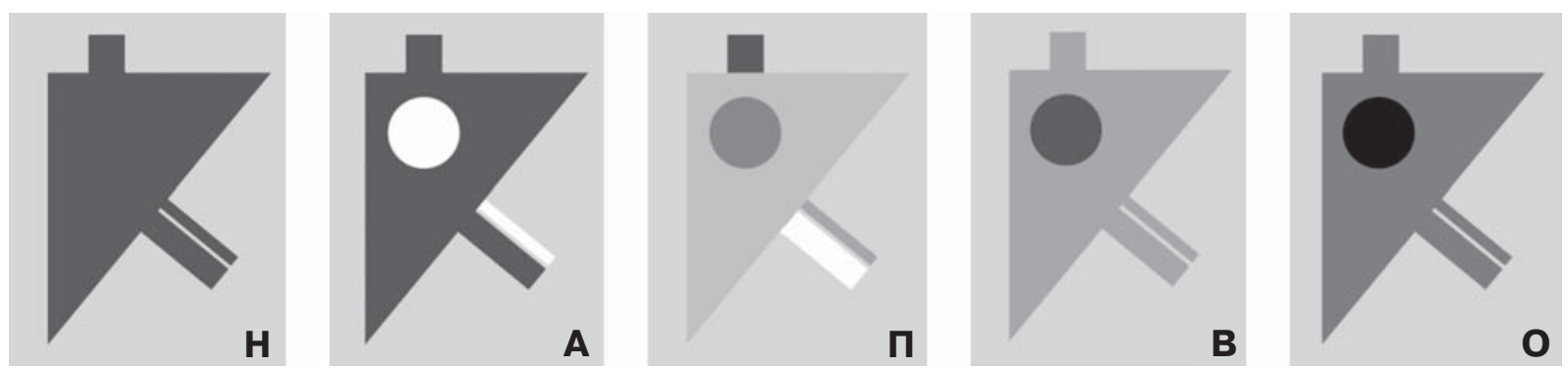

Рис. 1. Схематическое изображение типичного паттерна контрастирования ГЦР. В нативную фазу образование изоденсно паренхиме, что характерно для малых опухолей. В артериальную фазу происходит выраженное накопление контрастного препарата, а в последующие фазы - его вымывание. Фазы исследования: Н - нативная, А - артериальная, П - портальная, В - венозная, О - отсроченная.

Fig. 1. Schematic representation of a typical enhancement pattern of hepatocellular carcinoma. In the native phase the tumor density is equal to parenchymal density, which is typical for small nodes. In the arterial phase a massive accumulation of contrast agent occured and in the subsequent phases the contrast agent washout can be observed. Examination phases: $\mathrm{H}-$ native, $\mathrm{A}-$ arterial, $\Pi$ - portal, B - venous, $\mathrm{O}$ - delayed phases.
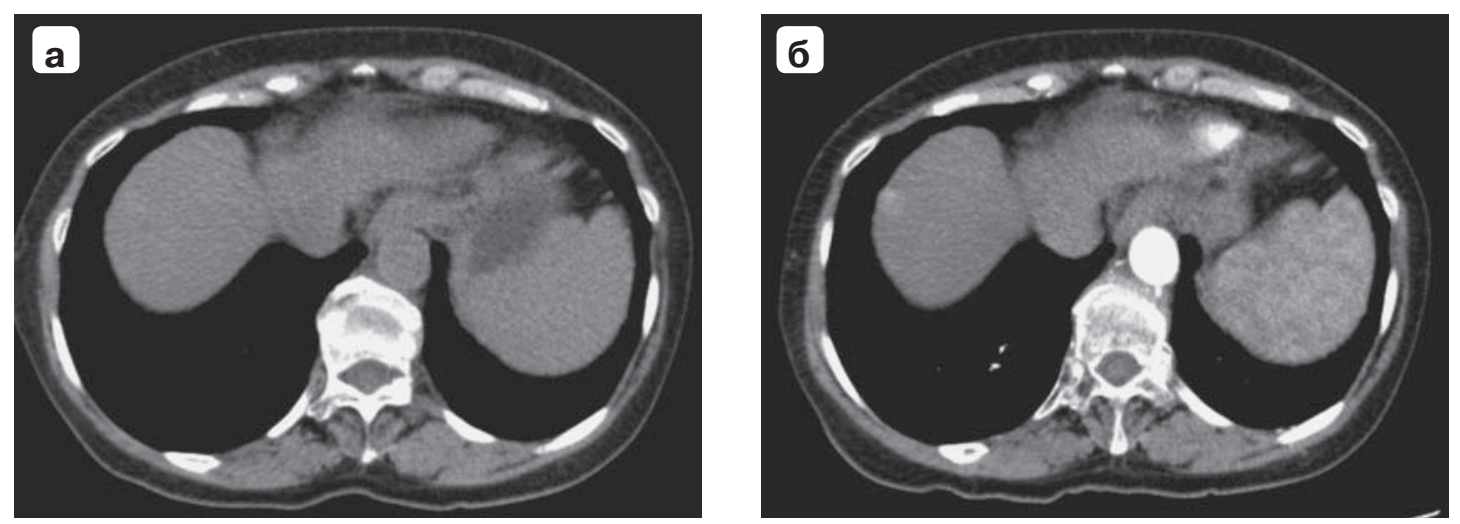

Рис. 2. Компьютерная томограмма. ГЦР, цирроз печени. Четырехфазное исследование: а - нативная фаза, опухоль не отличается по плотности от окружающей паренхимы, не видна; б, в - артериальная и портальная фаза, накопление контрастного препарата в опухоли VIII сегмента; г - отсроченная фаза, вымывание контрастного препарата. Уровень АФП 11000 нг/мл (норма 0-8 нг/мл).

Fig. 2. CT-scan of hepatocellular carcinoma, liver cirrhosis. Four-phase examination: a - native phase, the node density does not differ from the surrounding parenchyma, it is not; b, c - arterial phase, accumulation of contrast agent in a tumor of segment VIII; g - delayed phase, wash-out. 

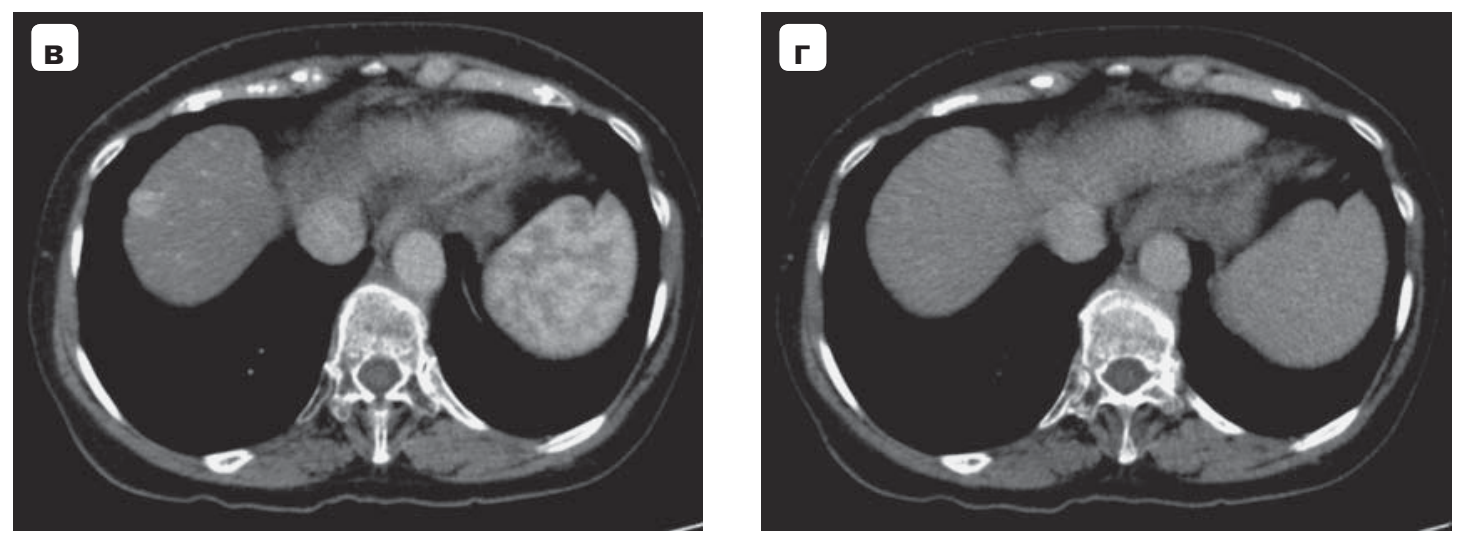

Рис. 2 (окончание). / Fig. 2 (end).
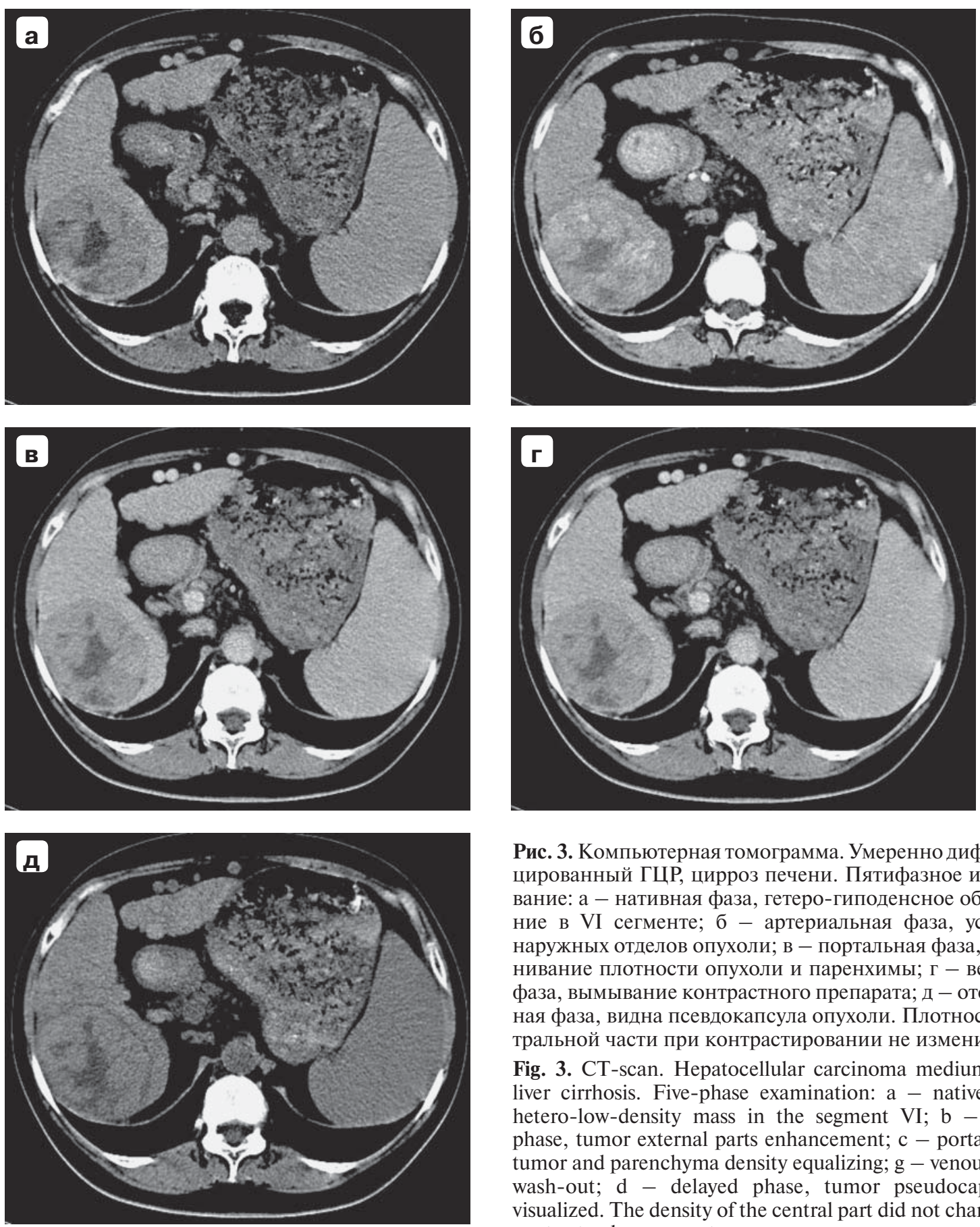

Рис. 3. Компьютерная томограмма. Умеренно дифференцированный ГЦР, цирроз печени. Пятифазное исследование: а - нативная фаза, гетеро-гиподенсное образование в VI сегменте; б - артериальная фаза, усиление наружных отделов опухоли; в - портальная фаза, выравнивание плотности опухоли и паренхимы; г - венозная фаза, вымывание контрастного препарата; д - отсроченная фаза, видна псевдокапсула опухоли. Плотность центральной части при контрастировании не изменилась.

Fig. 3. CT-scan. Hepatocellular carcinoma medium-grade, liver cirrhosis. Five-phase examination: a - native phase, hetero-low-density mass in the segment VI; b - arterial phase, tumor external parts enhancement; $\mathrm{c}$ - portal phase, tumor and parenchyma density equalizing; $\mathrm{g}$ - venous phase, wash-out; d - delayed phase, tumor pseudocapsule is visualized. The density of the central part did not change after contrast enhancememt. 

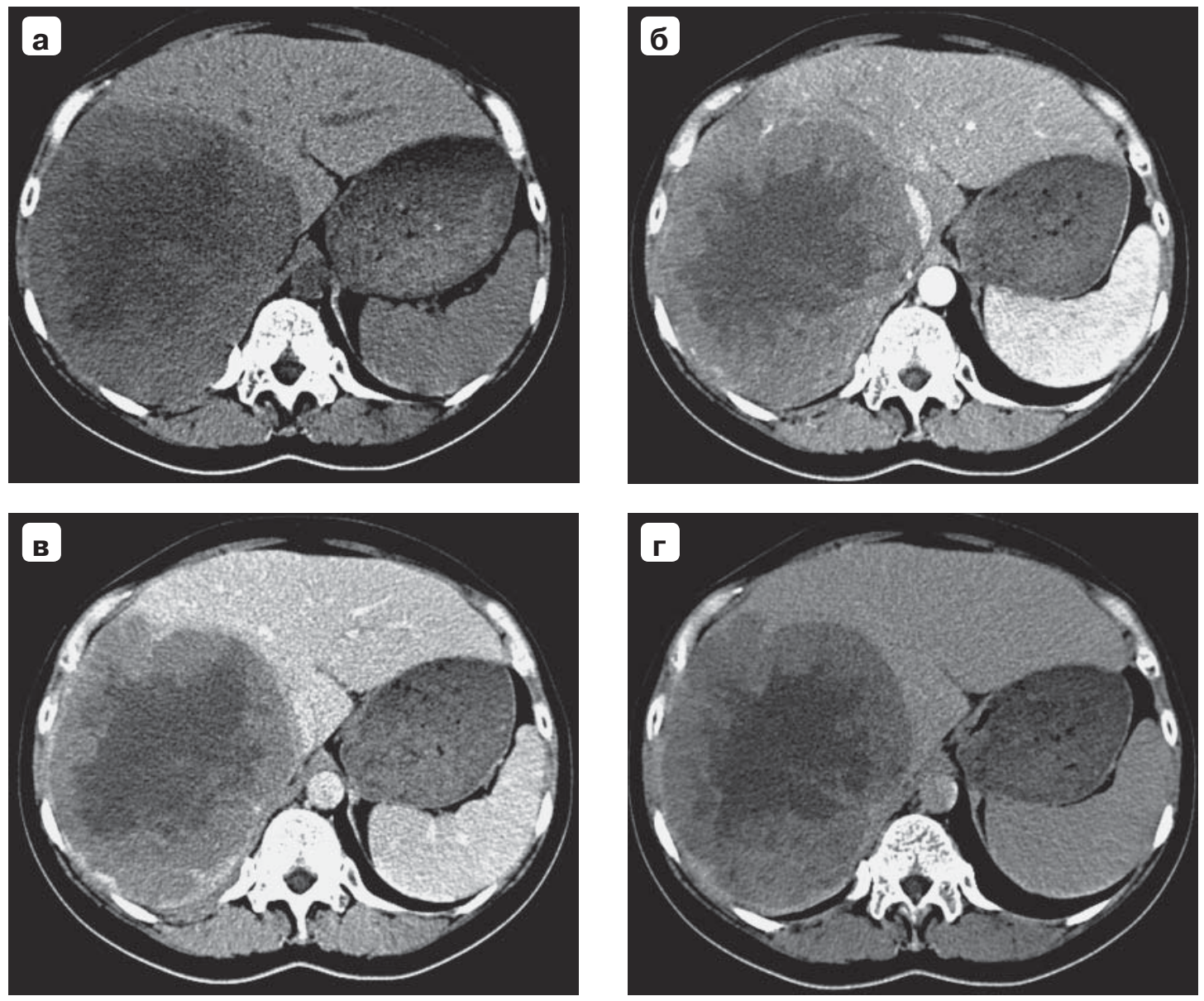

Рис. 4. Компьютерная томограмма. Низкодифференцированный ГЦР. Четырехфазное исследование: а - нативная фаза; б - артериальная фаза; в - портальная фаза; г - отсроченная фаза. Видна крупная опухоль в правой доле, обширная центральная зона некроза; капсула и гиперваскулярность в артериальную фазу отсутствуют. Клинических, лабораторных и КТ-признаков цирроза нет.

Fig. 4. CT-scan. Hepatocellular carcinoma low-grade. Four-phase examination; $a-$ native phase; $b$ - arterial phase; $c-$ portal phase; $d$ - delayed phase. A large tumor is visible in the right lobe, an extensive central zone of necrosis; capsule and hypervascularity in the arterial phase are absent. There are no clinical, laboratory, or CT signs of cirrhosis.
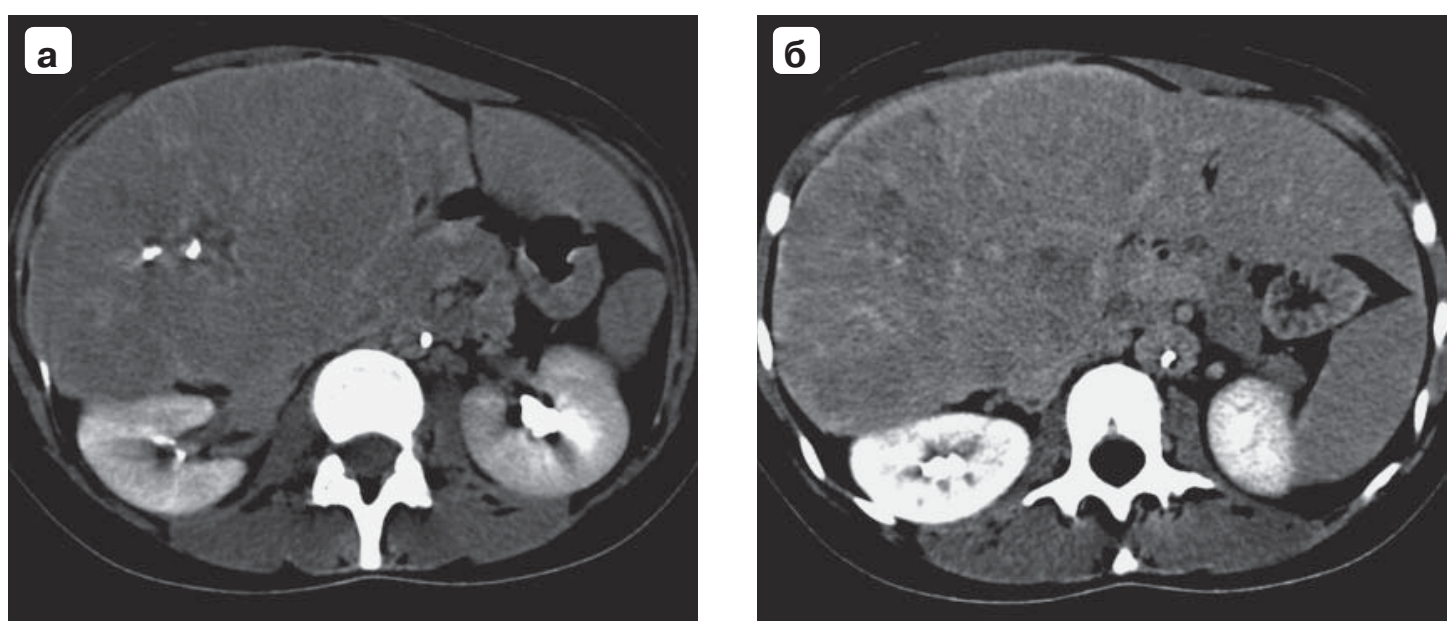

Рис. 5. КТ-артериограмма. Низкодифференцированный ГЦР: а - ранняя артериальная фаза, видны внутриопухолевые аневризмы; б - отсроченная фаза, видны участки кистозной дегенерации опухоли, контрастное усиление капсулы.

Fig. 5. CT-arteriogram. Hepatocellular carcinoma low-grade. a - early arterialphase; intratumoral aneurysms are visible; $\mathrm{b}$ - delayed phase; areas of cystic tumor degeneration and capsule contrast enhancement are visible. 

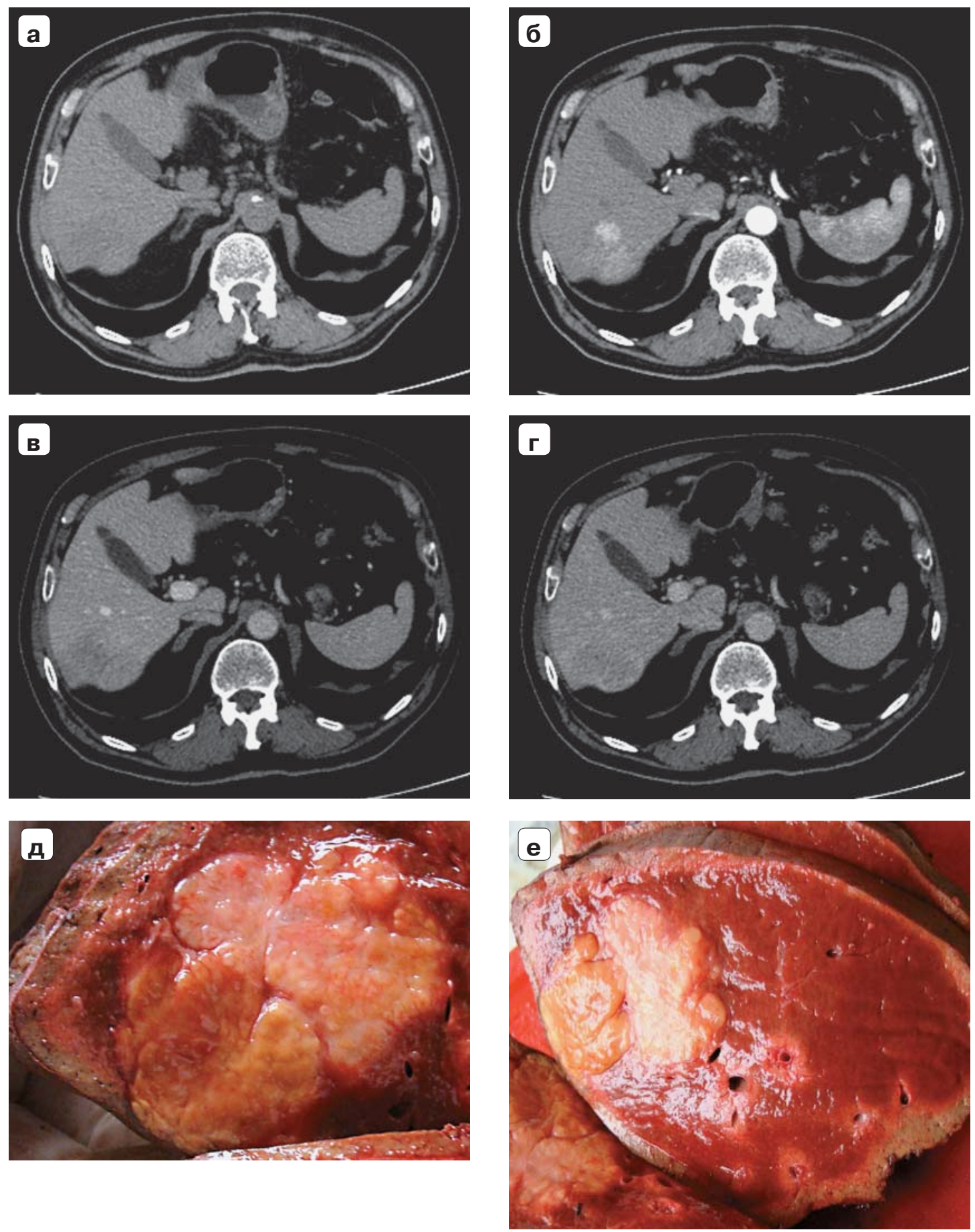

Рис. 6. Низкодифференцированный ГЦР: а - компьютерная томограмма, нативная фаза, гиподенсная опухоль задних сегментов правой доли; б - компьютерная томограмма, артериальная фаза, опухоль состоит из двух образований, контрастирующихся с различной интенсивностью, степень накопления контрастного препарата дорзальной опухолью существенно меньше, чем вентральной; в-г - компьютерная томограмма, портальная и отсроченная фаза, раннее вымывание контрастного препарата; д-е - макрофото, разный цвет опухолей обусловлен содержанием в них жира, инфильтрация дорзальной опухоли больше вентральной (подтверждено гистологически).

Fig. 6. Hepatocellular carcinoma low-grade: a - CT-scan, native phase, low-density tumor of the posterior segments of the right lobe; $b$ - CT-scan, arterial phase, tumor consists from two nodes with varying intensity contrast enhancement, the degree of the contrast agent accumulation by the dorsal tumor is significantly less than by ventral tumor; $\mathrm{c}-\mathrm{d}-\mathrm{CT}$-scan, portal and delayed phases, early wash-out; $\mathrm{e}-\mathrm{f}-$ macrophoto, the different color of tumors associated with the fat content in the tumor, the infiltration of the dorsal tumor is more than the ventral one (histologically confirmed). 

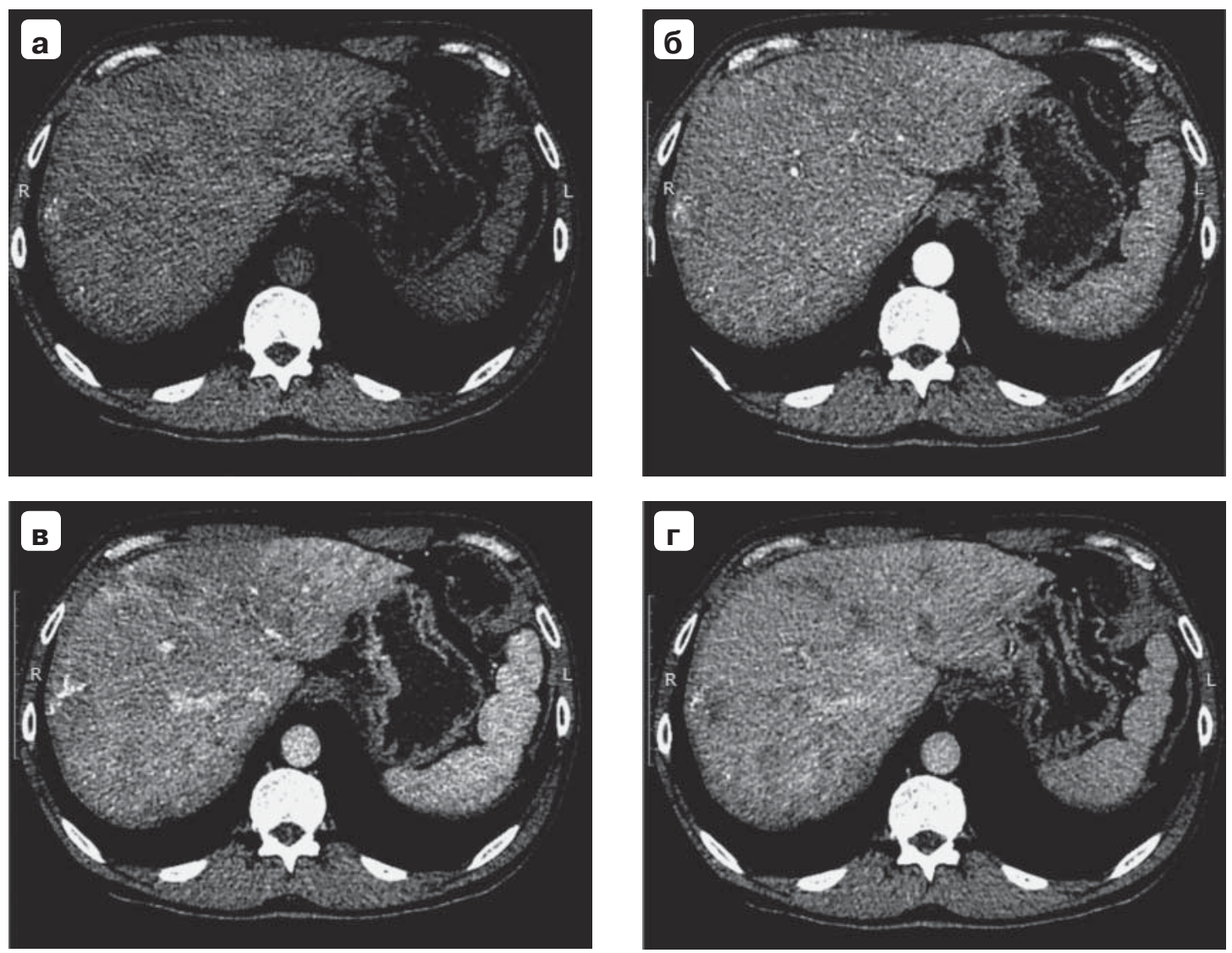

Рис. 7. Компьютерная томограмма. Низкодифференцированный ГЦР, осложненный портальным тромбозом. Четырехфазное исследование: а - нативная фаза; б - артериальная фаза; в - портальная фаза; г - отсроченная фаза. Диффузно-неоднородное изменение плотности паренхимы, негомогенное накопление контрастного вещества в бассейне тромбированной вены. Ни в одну из фаз контрастного усиления не удается отчетливо очертить опухолевый узел.

Fig. 7. CT-scan. Hepatocellular carcinoma low-grade, complicated with portal thrombosis. Four-phase examination: a - native phase, $\mathrm{b}$ - arterial phase, c - portal phase, $\mathrm{d}$ - delayed phase. Diffuse-heterogeneous parenchyma density change, in homogeneous accumulation of contrast agent in the thrombosed vein. There is not possible to clearly outline the tumor node to any one of the phases of contrast enhancement.
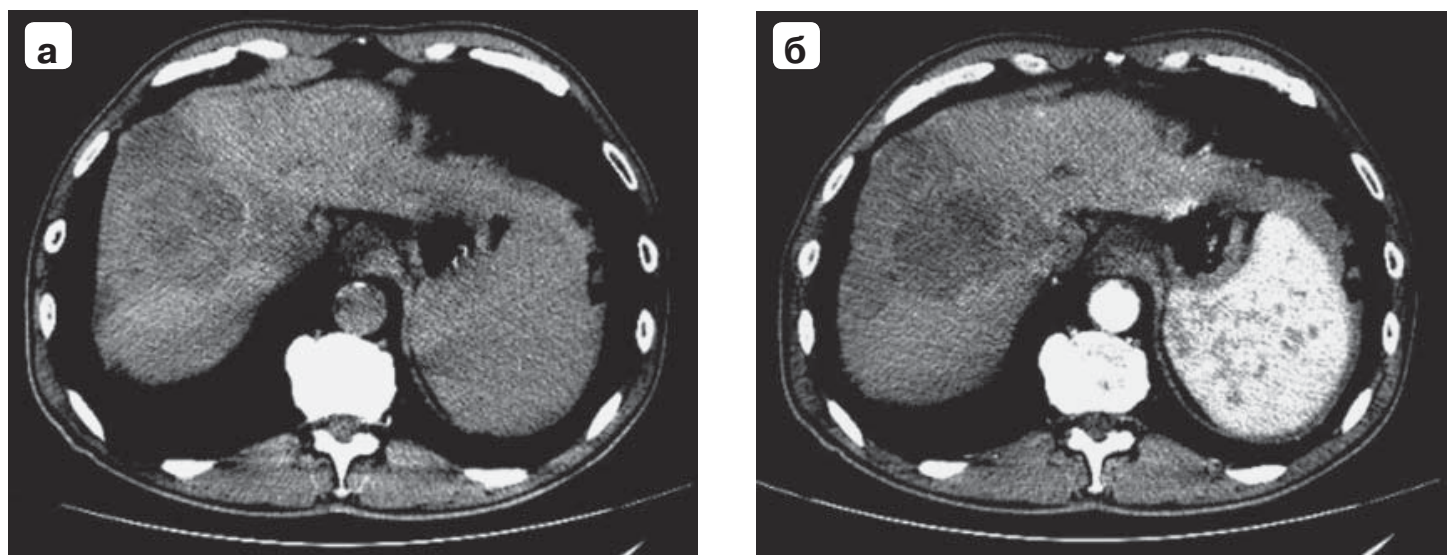

Рис. 8. Компьютерная томограмма. Низкодифференцированный ГЦР, осложненный портальным тромбозом, цирроз печени. Четырехфазное исследование: а, б - нативная и артериальная фаза, обнаруженный гетероденсный участок треугольной формы, окружающий опухоли, требует уточнения причин перфузионных изменений в сосудистых системах печени.

Fig. 8. CT-scan. Hepatocellular carcinoma low-grade, complicated with portal thrombosis, liver cirrhosis. Four-phase examination: $a, b-$ native and arterial phases, the detected heteroenhancet zone with triangular shape surrounding the tumors requires clarification of the reasons of perfusion changes in the vascular systems of the liver. 

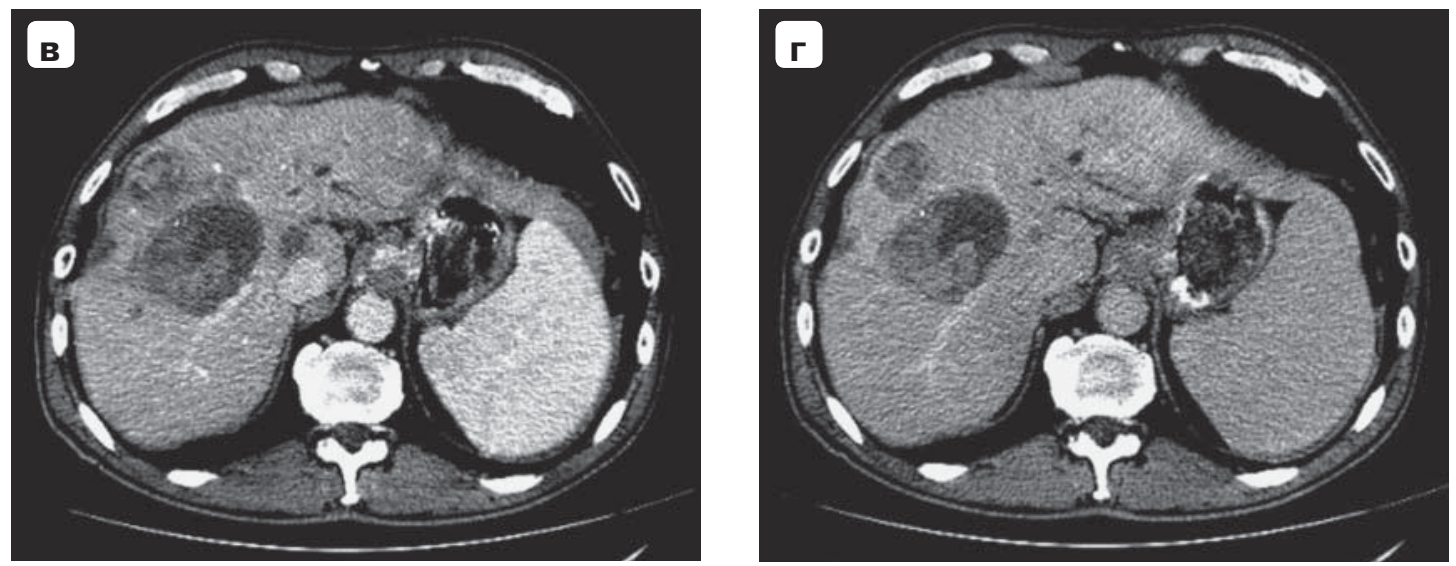

Рис. 8 (окончание). в - портальная фаза; г - отсроченная фаза. Некротические участки не накапливают контрастный препарат, но и в солидных зонах опухолей убедительного накопления не отмечено.

Fig. 8 (end). c - portal phase, d - delayed phase. Necrotic areas do not accumulate contrast agent, but there is no intensive accumulation in the solid parts of the tumor nodes.

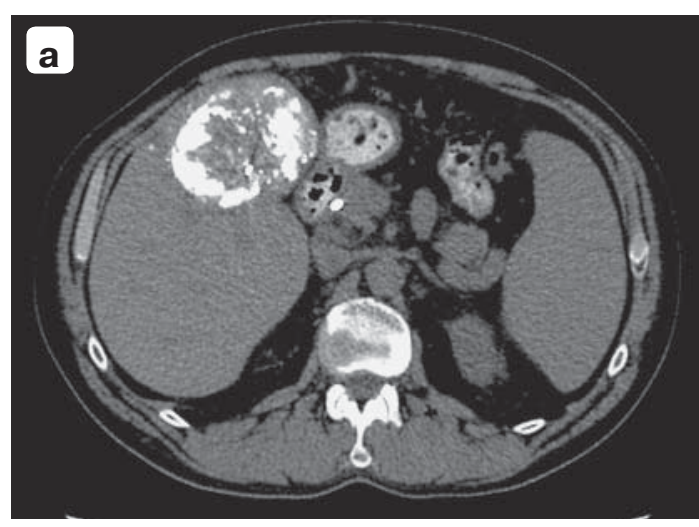

Рис. 9. Компьютерная томограмма. Умеренно дифференцированный ГЦР, 5-е сутки после внутриартериального введения взвеси химиопрепарата и липиодола: а - основная опухоль; б, в - множественные внутриорганные метастазы.

Fig. 9. CT-scan. Hepatocellular carcinoma medium-grade. The fifth day after intraarterial injection of a suspension of chemotherapeutic agent and lipiodol: $\mathrm{a}$ - the main tumor node; b, c multiple intra-organ metastases.
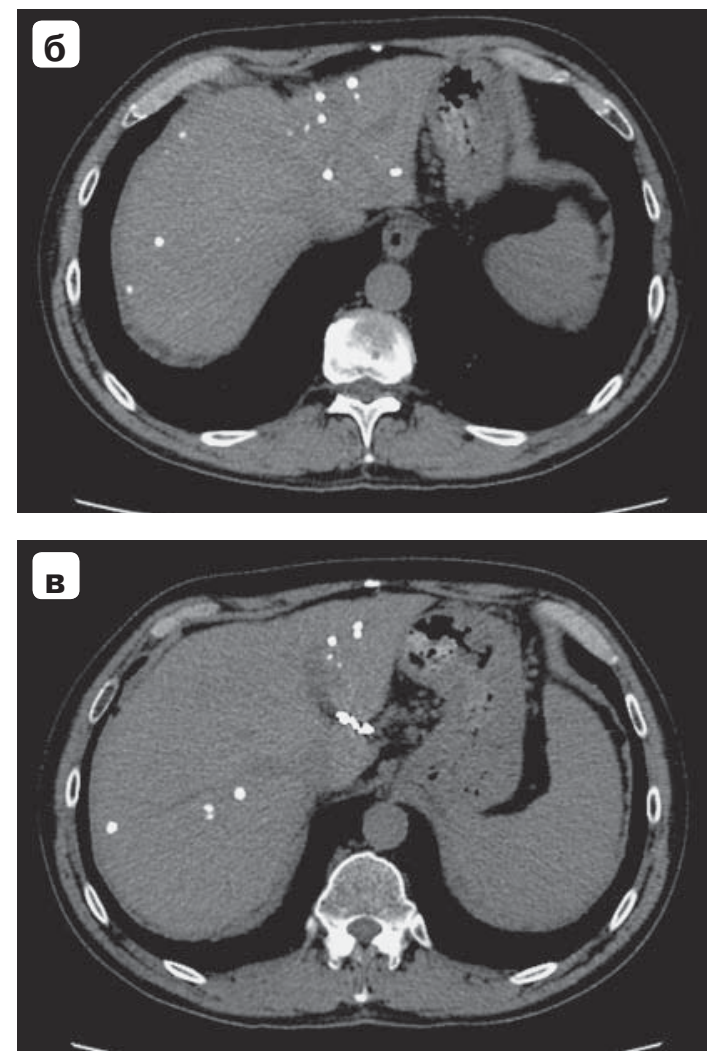

Особого внимания требует инфильтративный морфологический подтип ГЦР, при котором не формируется отчетливая опухоль, границы между пораженной и условно интактной (цирротически измененной) паренхимой стерты, рано возникает поражение портальных сосудов и опухолевый или коагуляционный портальный тромбоз. В связи с этим такой вариант ГЦР трудно диагностировать [14]. Собственное наблюдение приведено на рис. 7.

Несмотря на предсуществующий цирроз, вызывает трудности распознавание ГЦР, осложненного портальным тромбозом с гиповаскулярным паттерном контрастирования опухоли. 
Только у части пациентов можно выявить контрастирующееся узкое кольцо на периферии опухоли (см. рис. 8).

Большое значение в определении стадии опухолевого процесса имеет выявление внутриорганных метастазов. Высокая чувствительность химиоэмболизации печеночной артерии с липиодолом в обнаружении мелких сателлитных опухолей показана на рис. 9.

\section{Заключение}

Затруднения в лучевой диагностике ГЦР появляются при отклонении выявленного паттерна контрастирования от типичного. Разнообразие КТ-картины обусловлено многообразием морфологических вариантов ГЦР: макроскопической формой, степенью дифференцировки, содержанием жира, осложнениями.

\section{Участие авторов}

Розенгауз Е.В. - концепция и дизайн исследования, сбор и обработка материала, написание текста, редактирование.

Караханова А.Г. - концепция и дизайн исследования, сбор и обработка материала.

\section{Autors participation}

Rozengauz E.V. - concept and design of the study, collection and analysis of data, writing text, editing.

Karakhanova A.G. - concept and design of the study, collection and analysis of data.

\section{Список литературы}

1. Choi J.Y., Lee J.M., Sirlin C.B. CT and MR imaging diagnosis and staging of hepatocellular carcinoma: part I. Development, growth, and spread: key pathologic and imaging aspects. Radiology. 2014; 272 (3): 635-654.

https://doi.org/10.1148/radiol.14132361

2. Кармазановский Г.Г. Роль МСКТ и МРТ в диагностике очаговых заболеваний печени. Анналы хирургической гепатологии. 2019; 24 (4): 91-110.

https://doi.org/10.16931/1995-5464.2019491-110

3. Roberts L.R., Sirlin C.B., Zaiem F., Almasri J., Prokop L.J., Heimbach J.K., Murad M.H., Mohammed K. Imaging for the diagnosis of hepatocellular carcinoma: a systematic review and meta-analysis. Hepatology. 2018; 67 (1): 401-421.

https://doi.org/10.1002/hep.29487

4. Bruix J., Sherman M., Llovet J.M., Beaugrand M., Lencioni R., Burroughs A.K., Christensen E., Pagliaro L., Colombo M., Rodés J. EASL Panel of Experts on HCC. Clinical management of hepatocellular carcinoma.Conclusions of the Barcelona-2000 EASL conference. European Association for the Study of the Liver. J. Hepatol. 2001; 35 (3): 421-430. https://doi.org/10.1016/s0168-8278(01)00130-1

5. Lee J.H., Lee J.M., Kim S.J., Baek J.H., Yun S.H., Kim K.W., Han J.K., Choi B.I. Enhancement patterns of hepatocellular carcinomas on multiphasic multidetector row CT: comparison with pathological differentiation. Br. J. Radiol. 2012; 85 (1017): e573-e583. https://doi.org/10.1259/bjr/86767895
6. Туманова У.Н., Яшина Н.И., Кармазановский Г.Г., Щеголев А.И. Диагностические компьютерно-томографические критерии гепатоцеллюлярного рака разной степени дифференцировки в непораженной циррозом печени. Диагностическая и интервенционная радиология. 2015; 9 (1); 27-33.

7. Розенгауз Е.В., Караханова А.Г., Нестеров Д.В. Особенности паттерна контрастирования гепатоцеллюлярного рака при тромбозе воротной вены. Медицинская визуализация. 2019; 23 (4): 68-75. doi.org/10.24835/1607-0763-2019-4-68-75

8. Lafitte M., Laurent V., Soyer P., Ayav A., Balaj C., Petit I., Hossu G. MDCT features of hepatocellular carcinoma (HCC) in non-cirrhotic liver. Diagn. Interv. Imaging. 2016; 97 (3): 355-360. http://dx.doi.org/10.1016/j.diii.2015.09.007.

9. Basaran C., Karcaaltincaba M., Akata D., Karabulut N., Akinci D., Ozmen M., Akhan O. Fat-containing lesions of the liver: cross-sectional imaging findings with emphasis on MRI. AJR. Am. J. Roentgenol. 2005; 184: 1103-1110. https://doi.org/0361-803X/05/1844-1103

10. EASL-EORTC clinical practice guidelines: management of hepatocellular carcinoma. J. Hepatol. 2012; 56 (4): 908-943. https://doi.org/10.1016/j.jhep.2011.12.001.

11. Luca A., Caruso S., Milazzo M., Mamone G., Marrone G., Miraglia R., Maruzzelli L., Carollo V., Minervini M.I., Vizzini G., Gruttadauria S., Gridelli B. Multidetector-row computed tomography (MDCT) for the diagnosis of hepatocellular carcinoma in cirrhotic candidates for liver transplantation: prevalence of radiological vascular patterns and histological correlation with liver explants. Eur. Radiol. 2010; 20 (4): 898-907. https://doi.org/10.1007/s00330-009-1622-0

12. Kutami R., Nakashima Y., Nakashima O., Shiota K., Kojiro M. Pathomorphologic study on the mechanism of fat change in small hepatocellular carcinoma in humans. J. Hepatol. 2000; 33 (2): 282-289. https://doi.org/10.1016/s0168-8278(00)80369-4

13. Umar N.K., Badshah M.B., Sandrasegaran K., Ghabril M., Agarwal S., Tann M., Lacerda M., Kwo P.Y. Thepresens of portal vein thrombosis alters the classic enhancement associated with diagnosis of hepatocellular carcinoma. Dig. Dis. Sci. 2015; 60 (7): 2196-2200. https://doi.org/10.1007/s10620-015-3587-y.

14. Kneuertz P.J., Demirjian A., Firoozmand A., CoronaVillalobos C., Bhagat N., Herman J., Cameron A., Gurakar A., Cosgrove D., Choti M.A., Geschwind J.F., Kamel I.R., Pawlik T.M. Diffuse infiltrative hepatocellular carcinoma: assessment of presentation, treatment, and outcomes. Ann. Surg. Oncol. 2012; 19 (9): 2897-2907.

https://doi.org/10.1245/s10434-012-2336-0

\section{References}

1. Choi J.Y., Lee J.M., Sirlin C.B. CT and MR imaging diagnosis and staging of hepatocellular carcinoma: part I. Development, growth, and spread: key pathologic and imaging aspects. Radiology. 2014; 272 (3): 635-654.

https://doi.org/10.1148/radiol.14132361

2. Karmazanovsky G.G. The role of MDCT and MRI in the diagnosis of focal liver diseases. Annaly khirurgicheskoy gepatologii = Annals of HPB Surgery. 2019; 24 (4): 91-110. https://doi.org/10.16931/1995-5464.2019491-110 (In Russian)

3. Roberts L.R., Sirlin C.B., Zaiem F., Almasri J., Prokop L.J., Heimbach J.K., Murad M.H., Mohammed K. Imaging for the diagnosis of hepatocellular carcinoma: a systematic review and meta-analysis. Hepatology. 2018; 67 (1): 401-421.

https://doi.org/10.1002/hep.29487 
4. Bruix J., Sherman M., Llovet J.M., Beaugrand M., Lencioni R., Burroughs A.K., Christensen E., Pagliaro L., Colombo M., Rodés J. EASL Panel of Experts on HCC. Clinical management of hepatocellular carcinoma.Conclusions of the Barcelona-2000 EASL conference. European Association for the Study of the Liver. J. Hepatol. 2001; 35 (3): 421-430. https://doi.org/10.1016/s0168-8278(01)00130-1

5. Lee J.H., Lee J.M., Kim S.J., Baek J.H., Yun S.H., Kim K.W., Han J.K., Choi B.I. Enhancement patterns of hepatocellular carcinomas on multiphasic multidetector row CT: comparison with pathological differentiation. Br. J. Radiol. 2012; 85 (1017): e573-e583. https://doi.org/10.1259/bjr/86767895

6. Tumanova U.N., Yachina N.I., Karmazanovsky G.G., Shchegolev A.I. Computed tomography diagnostic criteria of hepatocellular carcinoma with varying degree of differentiation in the liver unaffected by cirrhosis. Diagnostic and interventional radiology. 2015; 9 (1); 27-33. (In Russian)

7. Rozengauz E.V., Karakhanova A.G., Nesterov D.V. Contrast enhancement patterns of hepatocellular carcinoma with portal vein thrombosis. Medical Visualization. 2019; 23 (4): 68-75. https://doi.org/10.24835/1607-0763-2019-4-68-75 (In Russian)

8. Lafitte M., Laurent V., Soyer P., Ayav A., Balaj C., Petit I., Hossu G. MDCT features of hepatocellular carcinoma (HCC) in non-cirrhotic liver. Diagn. Interv. Imaging. 2016; 97 (3): 355-360. http://dx.doi.org/10.1016/j.diii.2015.09.007.

9. Basaran C., Karcaaltincaba M., Akata D., Karabulut N., Akinci D., Ozmen M., Akhan O. Fat-containing lesions of the liver: cross-sectional imaging findings with emphasis on MRI.
AJR. Am. J. Roentgenol. 2005; 184: 1103-1110. https://doi.org/0361-803X/05/1844-1103

10. EASL-EORTC clinical practice guidelines: management of hepatocellular carcinoma. J. Hepatol. 2012; 56 (4): 908-943. https://doi.org/10.1016/j.jhep.2011.12.001.

11. Luca A., Caruso S., Milazzo M., Mamone G., Marrone G., Miraglia R., Maruzzelli L., Carollo V., Minervini M.I., Vizzini G., Gruttadauria S., Gridelli B. Multidetector-row computed tomography (MDCT) for the diagnosis of hepatocellular carcinoma in cirrhotic candidates for liver transplantation: prevalence of radiological vascular patterns and histological correlation with liver explants. Eur. Radiol. 2010; 20 (4): 898-907. https://doi.org/10.1007/s00330-009-1622-0

12. Kutami R., Nakashima Y., Nakashima O., Shiota K., Kojiro M. Pathomorphologic study on the mechanism of fat change in small hepatocellular carcinoma in humans. J. Hepatol. 2000; 33 (2): 282-289. https://doi.org/10.1016/s0168-8278(00)80369-4

13. Umar N.K., Badshah M.B., Sandrasegaran K., Ghabril M., Agarwal S., Tann M., Lacerda M., Kwo P.Y. Thepresens of portal vein thrombosis alters the classic enhancement associated with diagnosis of hepatocellular carcinoma. Dig. Dis. Sci. 2015; 60 (7): 2196-2200. https://doi.org/10.1007/s10620-015-3587-y.

14. Kneuertz P.J., Demirjian A., Firoozmand A., CoronaVillalobos C., Bhagat N., Herman J., Cameron A., Gurakar A., Cosgrove D., Choti M.A., Geschwind J.F., Kamel I.R., Pawlik T.M. Diffuse infiltrative hepatocellular carcinoma: assessment of presentation, treatment, and outcomes. Ann. Surg. Oncol. 2012; 19 (9): 2897-2907. https://doi.org/10.1245/s10434-012-2336-0

\section{Сведения об авторах [Authors info]}

Розенгауз Евгений Владимирович - доктор мед. наук, главный научный сотрудник отдела лучевой диагностики ФГБУ РНЦРХТ имени академика А.М. Гранова МЗ РФ, профессор кафедры лучевой диагностики и лучевой терапии ФГБОУ ВО СЗГМУ им. И.И. Мечникова МЗ РФ. https://orcid.org/0000-0003-1742-7783. E-mail: rozengaouz@yandex.ru Караханова Анна Геннадьевна - врач-рентгенолог отделения рентгеновской компьютерной томографии ФГБУ НМИЦ онкологии им. Н.Н. Петрова МЗ РФ. https://orcid.org/0000-0002-1913-9390. E-mail: annakarachanova@yandex.ru

Для корреспонденции *: Розенгауз Евгений Владимирович - 197758, Санкт-Петербург, пос. Песочный, ул. Ленинградская, д. 70, Российская Федерация. Тел.: 8-812-596-80-55. E-mail: rozengaouz@yandex.ru

Evgeny V. Rozengauz - Doct. of Sci. (Med.), Chief Researcher of the Department of Radiology, Granov Russian Scientific Center of Radiology and Surgical Technologies, Professor of the Department of Radiology and Radiation Therapy, North-Western State Medical University named after I. I. Mechnikov. https://orcid.org/0000-0003-1742-7783. E-mail: rozengaouz@yandex.ru

Anna G. Karakhanova - Radiologist of the Department of X-ray Computed Tomography, Petrov National Medical Research Center of Oncology. https://orcid.org/0000-0002-1913-9390. E-mail: annakarachanova@yandex.ru

For correspondence*: Evgeny V. Rozengauz - 70, Leningradskaya str. ,Pesochny, St. Petersburg, 197758, Russian Federation. Phone: 8-812-596-80-55. E-mail: rozengaouz@yandex.ru

Статья поступила в редакцию журнала 23.02.2020. Received 23 February 2020.
Принята к публикации 25.02.2020. Accepted for publication 25 February 2020. 